\title{
Autoimmune Diseases: An Introduction
}

\author{
Shubha Ratna Shakya \\ Department of Zoology, Amrit Campus, Tribhuvan University, Kathmandu, Nepal \\ For correspondence: shubharatnashakya@gmail.com
}

\begin{abstract}
The immune system recognizes and eliminates foreign agents, and protects the host against infection. Autoimmunity is a natural phenomenon whereself-reactive antibodies and autoimmune cells are present in all individuals. A combination of genetic predisposition and environmental factors contribute to the development of autoimmune diseases.Autoantibodies attack structures within individuals that produce them.Autoimmunity is a major cause for a number of serious and fatal diseases. Presence of one autoimmune disease increases the chance for simultaneously developing other autoimmune diseasesin the same person.
\end{abstract}

Key words: Antibodies, Immune system, Molecular mimicry, polygenic, self-reactive

\section{Introduction}

An immune system is a complex network of special cells and organs that defend the body from pathogens, such as viruses, bacteria, and parasites, and keeps us in a healthy state. The immune system has a unique ability to differentiate between self and non-self, i.e., it destroys the non-self proteins without attacking body's own (self) proteins. However, autoimmunity occurs to some degree in all individuals. When the immune system fails to differentiate between 'self' and 'non self', it can attack body's own proteins, thereby causing autoimmune disorders. Patients with autoimmune diseases frequently have unusual antibodies circulating in their blood that target their own body tissues. Autoimmune diseases can affect any part of the body, and have a myriad ofclinical manifestations that make diagnosis extremely difficult.Most autoimmune diseases disproportionately affect women. For diseases such as thyroiditis, scleroderma, lupus, and Sjögren's syndrome, more than 85 percent of patients are female.

Autoimmune disease is an illness that develops when an individual's immune system attacks its own body organs or tissues. The exact cause of autoimmune diseases is unknown, although some individuals are genetically more susceptible to developing autoimmune diseases than others. This mostly happens when certain genes start showing abnormalities. These genes could be of antibodies, T-cell receptors or major histocompatibility complex (MHC). In certain types of autoimmune diseases, such as rheumatic fever, a bacteria or virus may trigger an immune response and the antibodies or T-cells attack normal cells because the normal cellshave parts similar to that of an infecting germ (molecular mimicry).

Most autoimmune diseases are thought to be polygenic, involving more than one gene. Evidence from clinical reports suggests thatpatients with a family history of autoimmune diseases are more likely to develop an autoimmune disease. For example, patients with the autoimmune thyroid diseases, Graves' disease or Hashimoto's thyroiditis, have a family history of those diseases. Patients with autoimmune

Nepalese Journal of Zoology | Online Volume 3 Issue 1 | November 2015 | Page 63 
thyroid disease are also more likely to develop other autoimmune diseases such as systemic lupus erythematosus (lupus), pernicious anemia, type I diabetes or Addison disease.

External environmental factors such as hormones, diet, pesticides, drugs, toxins and/or infections are important in determining whether or not an individual will develop autoimmune diseases. Environmental agents are able to amplify autoimmunity in genetically susceptible individuals and to break tolerance in genetically resistant individuals, thereby increasing the risk of developing autoimmune disease. Creactive proteins (CRPs), produced by the liver, are an essential part of the immune system. The levels of CRPs are elevated in almost all autoimmune disorders. Since the whole immune system dysfunctions,a person suffering with an autoimmune disorder will show increased susceptibility to any bacterial infections.

Most autoimmune diseases are more common in women than in men. Conservative estimates indicate that nearly $80 \%$ of individuals with autoimmune diseases are women. Exceptions include diabetes mellitus, ankylosing spondylitis and inflammatory heart disease, which occur more frequently in men. Autoimmune diseasesare among the tenth leading causes of death among women, in all age groups up to 65 , in USA. No data is available for Nepal. It is predicted that estrogen inwomen may influence the immune system to predispose them to autoimmune diseases. Furthermore, presence of one autoimmune disease increases the chance for simultaneously developing other autoimmune diseases in the same person.

\section{Some Common Autoimmune Diseases}

Acute disseminated encephalomyelitis (ADEM) (or acute demyelinating encephalomyelitis): ADEM is a rare autoimmune disease marked by a sudden and widespread attack of inflammation in brain and spinal cord due to viralinfection. ADEM also attacks nerves of the central nervous system and damages their myelin insulation, thereby destroying the white matter. ADEM's symptoms resemble that of multiple sclerosis (MS), therefore the disease is classifiedas a multiple sclerosis borderline disease. However, ADEM has several features that are different from MS. Unlike MS, ADEM occurs mostly in children and is marked by rapid fever. ADEM leads to loss of consciousness, coma and death, which is very rare in MS (except for severe MS cases).

Multiple Sclerosis: Multiple sclerosis (MS), also known as disseminated sclerosis or encephalomyelitisdisseminata, is the most common autoimmune disorder affecting the central nervous system. The name multiple sclerosis refers to scars (sclerae-better known as plaques or lesions) in the white matterof the brain and spinal cord. It is a demyelinating disease in which the insulating coverofnerve cells in the brain and spinal cord are damaged. This damage disrupts the ability to communicate, resulting in a wide range of signs and symptoms, including physical, mental, and sometimes psychiatric problems. There is no known cure for multiple sclerosis.

The cause of MS is still unknown. Scientists believe that the disease is triggered by unidentified environmental factor(s) in a person who is genetically predisposed to MS. In multiple sclerosis (MS), myelin sheaths around nerve fibers of the central nervous system (CNS) are damaged. Such damage interferes with transmission of nerve signals between the brain, spinal cord and the rest of the body. The myelin sheath is to a nerve cell what wire insulation is to a wire. Both help to conduct electrical signals better. Disrupted nerve signals result in walking problems, numbness, and balance issues. Depending on where the damage occurs, symptoms of MS vary from one person to another, and vary over time for any given individual.

Nepalese Journal of Zoology | Online Volume 3 Issue 1 | November 2015 | Page 64 
Ankylosing spondylitis: It is an inflammatory disease that can cause some of the spine vertebrae to fuse together. This fusing makes the spine less flexible and can result in a hunched-forward posture. If ribs are affected, it may cause difficulty in taking deep breaths. Ankylosing spondylitis mostly affects men. There is no cure for ankylosing spondylitis, but treatments can decrease pain and lessen symptoms.

Hashimoto's thyroiditis (HT): Hashimoto's thyroiditis is an autoimmune disease that affects the thyroid. Thyroid is an important endocrine glandlocated in front of the neck. It secretes thyroxine (T4) and triiodothyronine (T3) hormones that regulate growth and many important reactions that use energy. Thyroid hormone levels are controlled by thyroid stimulating hormone (TSH), which is secreted by the pituitary gland. With Hashimoto's disease, the immune system makes auto-antibodies that damage thyroid cells and interfere with their ability to make thyroid hormone. Over time, thyroid damage can lead tolow thyroid hormone levels. This is called an underactive thyroid or hypothyroidism. An underactive thyroid slows downvarious functions of the body such as heart rate, brain function, and the rate your body turns food into energy. Hashimoto's disease is the most common cause of an underactive thyroid. An enlarged thyroid, called a goiter, is often the first sign of Hashimoto's disease. The goiter may cause the front of the neck to look swollen. If large, it may cause a feeling of fullness in the throat or make it hard to swallow. It rarely causes pain. Hashimoto's disease is more common in women than in men.

Systemic lupus erythematosus (SLE): SLE is an autoimmune disease that attacks the brain, kidneys, joints, skin, heart, lungs and other organs in the body. People with this problem sometimes have a characteristic 'malar rash' on their face. Auto-antibodies produced in this disease attack a vast array of tissue antigens such as DNA, histones, RBCs, platelets, leukocytes and clotting factors. Interaction of these auto-antibodies with their specific antigens produces various disease symptoms. Ultraviolet radiation from sun exposure can exacerbate disease in patients with systemic lupus erythematosus.

Graves' disease(GD): Graves' disease is an autoimmune disease that affects the thyroid, a small gland situated in front of the neck. It makes hormones called T3 and T4 that regulate the body's usage of energy. Production of thyroid hormones is regulated by the thyroid-stimulating hormone (TSH), which is secreted by the pituitary (a pea-sized gland in the brain). In Graves' disease, the immune system makes autoantibodies that act like TSH, causing the thyroid to make more thyroid hormone than the body needs. This is called an overactive thyroid or hyperthyroidism. An overactive thyroid speeds up every function of the body such as heart rate and metabolism. Graves' disease is one cause of hyperthyroidism. Unlike other causes of an overactive thyroid, Graves' disease can also cause eye changes. For some people with Graves' disease, tissues behind the eyes become inflamed and swollen, causingone or both eyeballs to bulge out of the head and sometimes affects vision.

Both men and women can get Graves' disease, but women are 10 times more affected than men. Graves' disease occurs in people of all ages, but most often starts in between ages 20s and 30s. People who get Graves' disease often have family members with thyroid or other autoimmune diseases.

Myasthenia gravis (MG):Myasthenia gravis, also known as Goldflam disease, is an autoimmune neuromuscular disease characterized by varying degrees of weakness of the skeletal (voluntary) muscles. In Myasthenia gravis, literally meaning 'grave muscle weakness,' the muscles become easily tired and weak because there is a problem with transmission of nerve impulses to muscles. It occurs when normal communication between nerve and muscle is interrupted at the neuromuscular junction the place where nerve cells connectto the muscles they control. Normally when impulses travel down

Nepalese Journal of Zoology | Online Volume 3 Issue 1 | November 2015 | Page 65 
the nerve, the nerve endings release a neurotransmitter called acetylcholine. Acetylcholine travels from the neuromuscular junction and binds to acetylcholine receptors to activate and generate a muscle contraction. In MG,circulating abnormal antibodies block, alter, or destroy acetylcholine receptors at the post-synaptic neuromuscular junction, thereby preventing muscle contraction. These antibodies are produced by the body's own immune system.In MG, certain muscles that control eye and eyelid movement, facial expression, chewing, talking, swallowing and breathing are often, but not always, affected. Muscles that control neck and limb movements may also be affected, however, cardiac muscles are not affected. The disease is treated with immunosuppressant or cholinesterase inhibitors. Although there is no cure, treatment is effective in alleviating symptoms of arm/leg weakness, double vision, drooping eyelids, speech difficulties, chewing, swallowing and breathing. In fact, many people with MG become completely free of symptoms after treatment. Itis more common in women under 40 years and men over 60 . However, it may affect individuals of any age.

Diabetes type 1: Diabetes type $I$ isone of the most common autoimmune disorders in many countries of the world including Nepal. It is aninsulin-dependent diabetes. Insulin hormone, secreted by beta cells of Islets of Langerhans in the pancreas, controls blood glucose level. The immune system targets and ultimately destroys these insulin producing beta cells, causing insulin deficiency. At the same time, B cells make antibodies against beta cell proteins. When the number of beta cells is reduced by approximately $80 \%$, the body is unable to secrete enough insulin, thereby causing an elevated blood glucose level. High blood sugar, clinically diagnosed as diabetes, can damage eyes, kidneys, nerves, gums and teeth. But, the most serious problem caused by diabetes is heart disease.

Rheumatoid arthritis (RA): Rheumatoid arthritis is a common autoimmune disorderin which the auto-antibodies called rheumatoid factors attack joint liningsthroughout the body. Bodyjointssuch as knee or finger joints are attacked and destroyed. People with rheumatoid arthritis have joint pain and face difficulty while moving.

Goodpasture syndrome (GS): Goodpasture's disease (also known as Goodpasture Syndrome, antiglomerular basement membrane disease, anti-GBM disease) is an autoimmune disorder. It was first described by Ernest Goodpasture in 1919. The GS syndrome results when the immune system attacks a particular molecule, the Goodpasture antigen, found in the kidneys and the lungs.Persons with this syndrome develop antibodies that attack a protein called collagen found in tiny air sacs in the lungs and in filtering units (glomeruli) of the kidneys.These antibodies are called anti-glomerular basement membrane antibodies. The glomerular basement membrane, part of the kidneys, helps filter waste and extra fluid from the blood. Anti-glomerular basement membrane antibodies are antibodies against this membrane. They damage the basement membrane and cause rapid destruction of kidneys.Sometimes this disorder is triggered by a viral respiratory infection or by breathing in hydrocarbon solvents. In such cases, the immune system may attack organs or tissues because it mistakes them for these viruses or foreign chemicals.

AntiphospholipidSyndrome(APS):Antiphospholipid Syndrome, also known as Antiphospholipid Antibody Syndrome (APLS), Hughes Syndrome, or Sticky Blood, is an immune disorder in which the person's immune system produces antibodies called antiphospholipid antibodies. The abnormal antibodiesattack proteins and fats in the blood, specifically phospholipids. Such attacksmake the blood excessively sticky and results in a significantly higher risk of developing blood clots.

In APS, thromboses (abnormal blood clots) can develop in any blood vessel of the body, but it is most frequent in the veins and arteries (arterial and venous thrombosis) of the lower limbs. If a blood clot forms in the vessels of the brain, blood flow is impaired and can lead to stroke. Approximately 1 in

Nepalese Journal of Zoology | Online Volume 3 Issue 1 | November 2015 | Page 66 
every 3 patients with APS has heart valve abnormalities. Patients most commonly develop DVT (deep vein thrombosis) - blood clots that form in the legs; however, clots may also form in the kidneys, lungs and other organs.

Guillain-Barre Syndrome: The immune system attacks nerves that connect the brain and spinal cord with rest of the body. Damage to these nerves make it difficult to transmit signals, as a result, the muscles have trouble responding to the brain.Guillain-Barré syndrome has been associated with a number of bacterial and viral infections.

Vitiligo: Vitiligo, a common autoimmune disorder, is characterized by patchy loss of pigment in the skin and hair. The immune system destroys cells that give skin its color. It can also affect tissue inside the mouth and nose.Vitiligo often clusters in families, and is frequently seen in individuals who have multiple autoimmune diseases.

Addison's disease: Addison's disease is a disorder that occurs when outer layer of the adrenal glands produce insufficient amounts of cortisolhormone. This occurs when the body's immune system mistakenly attacks the adrenal glands. Addison's disease is alsocalled "adrenal insufficiency" or hypocortisolism. Cortisol helps the body respond to stress, regulates body's use of protein, carbohydrates and fat, maintains blood pressure and cardiovascular function, and controls inflammation. Addison's disease can be life threatening, and can occur in all age groups and affect both sexes.

Aldosterone regulates the amount of salt and water in the body, which is the main way to regulate blood volume and to control blood pressure. When aldosterone levels are low, kidneys cannot keep salt and water levels in balance, thereby causing low blood pressure.

\section{Risk Factors}

Autoimmune diseases are a leading cause of death and disability. Some autoimmune diseases are rare, while others, such as Hashimoto's disease, are common. Autoimmune diseases can affect anyone, but people who might be at greater risk are as follows:

- Women of childbearing age: More women than men have autoimmune diseases, which often start during their childbearing years.

- People with family history: Some autoimmune diseases run in families, such as lupus and multiple sclerosis. It is also common for different types of autoimmune diseases to affect different members of a single family. Inheriting certain genes can make it more likely to get an autoimmune disease. But, a combination of genes and other factors may trigger the onset of a disease.

- People and their environment: Certain events or environmental exposures may cause some autoimmune diseases or make them worse. Sunlight, chemicals called solvents, and viral and bacterial infections are linked to many autoimmune diseases.

- People of certain races or ethnic backgrounds: Some autoimmune diseases are more common among certain groups of people. For instance, type 1 diabetes is more common in white people. Lupus is more severe in African-American and Hispanic people.

Although each disease is unique, many share common symptoms such as fatigue, dizziness and low-grade fever. For many autoimmune diseases, symptoms come and go, and can be mild or severe. When

Nepalese Journal of Zoology | Online Volume 3 Issue 1 | November 2015 | Page 67 
symptoms do not appear for some time, it's called remission. Flares are the sudden and severe onset of symptoms.

\section{Treatments}

There are many types of medicines used to treat autoimmune diseases. Here is a list of available treatments for autoimmune diseases:

Relieve symptoms:Drugs like aspirin and ibuprofen can be used for mild symptoms. Severe symptoms may need prescription drugs to help relieve pain, swelling, depression, anxiety, sleep problems, fatigue, or rashes.

Replace vital substances the body can no longer make on its own: Some autoimmune diseases, like type 1 diabetes mellitus and thyroid disease, can affect the body's ability to make substances that are essential for its function. For example, pancreatic islet cells of type 1 diabetes mellitus patients cannot produce insulin, therefore patients can take insulin to regulate blood sugar. Similarly, patients with autoimmune thyroiditis can be treated with thyroid hormones to restore thyroid hormone levels. These methods do not halt the autoimmune process, although patients may undergo remission while receiving symptom-based treatment. In most cases, however, patients must depend on replacement therapy throughout his or her lifetime. Sometimes a damaged organ can be replaced by transplantation. For example, scientists are now testing the effectiveness of islet cell transplant as a treatment for diabetes.

Use of immunosuppressants: Some immunosuppressive drugs can suppress auto-immune response and ameliorate disease manifestations. However, because these drugs also reduce the individual's resistance to infection, they must be used with great caution. Additionally, they often have adverse side effects. Such treatments are most often used for debilitating diseases such as lupus and rheumatoid arthritis. These treatments provide non-specific suppression of the immune system, and do not distinguish between a pathologic autoimmune response and a protective immune response. Immunosuppressive drugs such asazathioprine, ayclosporin and corticosteroids are often given to reduce severity of autoimmune disorders.Azathioprine (Imuran) is not only used to prevent organ rejection in kidney transplants, but also in treatment of rheumatoid arthritis. Cyclosporin (Sandimmune, Neoral) is usedfor multiple sclerosis, diabetes and myesthenia gravis.Mycopehnolate is used to prevent kidney problems associated with lupus erythematosus.

Plasmapheresis: Plasmapheresis is a process in which blood is separated into cells and plasma (liquid) by centrifugation. The plasma components such as autoantibodies, which can cause autoimmune disease, are removed.

T-cell vaccination: Vaccination using $\mathrm{T}$-cells prevent activation of autoreactive $\mathrm{T}$-cells. It is an effectiveway of treating autoimmune diseases.

Monoclonal antibodies: Monoclonal antibodies decrease T or B cells, act on activated T cells, inhibit particular cytokine mediators of inflammation, or block recruitment and localization of lymphocytes to the target organ. Although these targeted approaches usually have fewer side effects, they may increase a patient's vulnerability to infection, and therefore must be used with caution.

Stem cells: Adult hematopoietic stem cell transplantation can be done.Stem cell therapies might allow replacement or repair of damaged organs.

\section{Prevention}

Nepalese Journal of Zoology | Online Volume 3 Issue 1 | November 2015 | Page 68 
A person with an autoimmune disease can follow these steps to stay healthy:

- Eat healthy and well-balanced meals:Diet should include fruits and vegetables, whole grains, fat-free or low-fat milk products, and lean source of proteins. Saturated fat, trans fat, cholesterol, salt, and added sugars must be limited. Healthy and well balanced meals provide all the required nutrients for the body.

- Get regular physical activity: A gradual and gentle exercise program often works well for people with long-lasting muscle and joint pain. Yoga or tai chi exercises may be helpful.

- Get enough rest: Rest allows body tissues and joints to repair. Sleeping is a great way to help both body and mind. If you don't get enough sleep, your stress level and your symptoms could get worse. Most people need at least 7 to 9 hours of sleep each day to feel well-rested. Better sleep helps to tackle problems better and lowers risk for illness.

- Reduce stress:Stress and anxiety can also trigger symptoms of some autoimmune diseases. So,lead asimple life and cope with daily stressors. Meditation, self-hypnosis, and guided imagery are simple relaxation techniques that might help to reduce stress and lessen pain.

\section{Conclusion}

Fundamentally, all autoimmune diseases are a consequence of impaired immune function that results from interactions of genetic and environmental factors. These diseases pose a significant challenge to the field of immunology. They are different from other diseases, as our own protein becomes our foe. The treatment of autoimmune diseases is equally challenging as the degree of severity and symptoms vary from person to person. There is no cure for autoimmune diseases, but treatments can decrease pain and lessen symptoms. Furthermore, most autoimmune diseases are chronic in nature, requiring a lifetime of care. It is necessary to understandthe mechanisms behindderegulation of immune responses to develop better therapies for treatment and possibly even for prevention ofautoimmune diseases.

\section{References}

Abbas, A. K., Lohr, J., Knoechel, B. andNagabhushanam, V. 2004.T cell tolerance and autoimmunity. Autoimmunity Reviews, 3: 471-475.

Dale, R. C. 2003. "Acute disseminated encephalomyelitis". SeminPediatr Infect Dis, 14 (2): 90-5.

De Groot, P. G., Meijers, J. C. and Urbanus, R. T. 2012. Recent developments in our understanding of the antiphospholipid syndrome. Int J Lab Hematol,34 (3):223-31.

Fairweather, D. and Rose N. R. 2004.Women and autoimmune diseases.Emerging. Infectious Diseases, 10: 2005-2011.

Fairweather, D. and Rose, N. R. 2005. Inflammatory heart disease: a role for cytokines. Lupus 14 (9): 646-651.

Feldmann, M. andMaini, R. N. 2003.Lasker clinical medical research award. TNF defined as a therapeutic target for rheumatoid arthritis and other autoimmune diseases. Nature Medicine,9: 12451250.

Garg, R. K. 2003. "Acute disseminated encephalomyelitis". Postgrad Med J, 79 (927): 11-7.

Goodnow, C. C., Sprent, J., Fazekas de St.Groth, B. andVinuesa, C. G. 2005.Cellular and genetic mechanisms of self-tolerance and autoimmunity.Nature,435: 590-597.

Nepalese Journal of Zoology| Online Volume 3 Issue 1 | November 2015 | Page 69 
Huynh, W., Cordato, D. J., Kehdi, E., Masters, L.T. andDedousis, C. 2008. "Post-vaccination encephalomyelitis: literature review and illustrative case". J ClinNeurosci,15 (12): 1315-1322.

Jones, C. T. 2003. "Childhood autoimmune neurologic diseases of the central nervous system".NeurolClin, 21 (4): 745-64.

Nelson, B. H. 2004. IL-2, regulatory T cells, and tolerance. Journal of Immunology, 172: 3983-3988.

Rose, N. R and Mackay, I. R. (eds) 2006. The Autoimmune Diseases, $4^{\text {th }}$ edn. London: Elsevier Academic Press. 